International Mathematical Forum, 1, 2006, no. 19, 909-932

\title{
The Existence of Two New Classes of Near-Skolem Type Sequences
}

\author{
Colin Reid and Nabil Shalaby \\ Department of Mathematics and Statistics \\ Memorial University of Newfoundland \\ St.John's, Newfoundland, Canada, A1C 5S7
}

\begin{abstract}
Let $m, n$ be two positive integers, $m \leq n$. An $m$-near-Skolem sequence of order $n$ and defect $m$ is a sequence $m-S_{n}=\left(s_{1}, s_{2}, \cdots, s_{2 n-2}\right)$ of $2 n-2$ non-negative integers such that the following conditions hold:

1. for each $k \in\{1,2, \cdots, m-1, m+1, \cdots, n\}$ there exist exactly two elements $s_{i}, s_{j} \in m-S_{n}$ such that $s_{i}=s_{j}=k$

2. if $s_{i}=s_{j}=k$, then $|i-j|=k$

For example, $(4,1,1,2,4,2)$ is an example of a 3 -near-Skolem sequence of order 4.

In this article we prove that the necessary conditions are also sufficient for the existence of two new classes of near-Skolem type sequences; one with a hook two places from the last entry of the sequence and one with a hook two places and one place from the last entry. The sequences $(6,3,1,1,3,5,6,2,0,2,5)$ and $(6,2,5,2,1,1,6,5,3,0,0,3)$ are examples.
\end{abstract}

\section{Mathematics Subject Classification: 05B}

Keywords: Skolem, Langford and Rosa sequences, near-Skolem type sequences, Designs.

\section{Introduction}

The problem to be discussed in this article can be traced back to the work of Th. Skolem. In 1957 ([21]), while studying Steiner triple systems, Th. Skolem considered partitioning the integers $\{1,2, \cdots, 2 n\}$ into $n$ pairs $\left(a_{i}, b_{i}\right)$ such that $\left|a_{i}-b_{i}\right|=i$ for all $1 \leq i \leq n$. He showed that such a partition exists if and only if $n \equiv 0,1(\bmod 4)$. For example, for $n=4$, we get the partition $(1,5),(2,4),(3,6),(7,8)$. These partitions were later written as a sequence by Nickerson ([10]). For example, the above partition may be written as the 
sequence $(4,2,3,2,4,3,1,1)$. These sequences are now referred to as Skolem sequences of order $n$.

Skolem also considered partitioning the integers $\{1,2, \cdots, 2 n-1,2 n+1\}$ into $n$ pairs $\left(a_{i}, b_{i}\right)$ such that $\left|a_{i}-b_{i}\right|=i$ for all $1 \leq i \leq n$. When such a partition is written in Nickerson's form, we need to place a 0 , or "hook", in the second last position of the sequence. For example, for $n=3$, the partition $(1,4),(2,3),(5,7)$ gives us the sequence $(3,1,1,3,2,0,2)$. These sequences are known as hooked Skolem sequences of order $n$. O'Keefe, [12], showed that such a sequence exists if and only if $n \equiv 2,3(\bmod 4)$. A more general form of a hooked Skolem sequence is an extended Skolem sequence of order $n$. In this sequence, the hook may occur anywhere. In [2], Baker showed that the necessary conditions for the existence of this sequence were also sufficient.

In [22], Stanton and Goulden used a pairing concept for the purpose of constructing cyclic Steiner triple systems. In that paper they introduced the notation $P(1, n) / m$, which denotes a partition of the integers $\{1,2, \cdots, 2 n-2\}$ into $n-1$ pairs such that each of the integers $\{1,2, \cdots, m-1, m+1, \cdots, n\}$ appears exactly once as a difference amongst the pairs. When written in Nickerson's format, these partitions are known as m-near-Skolem sequences of order $n$ and defect $m$. Billington, [4], introduced similar notation, $P^{2}(1, n) / m-$ $\{j, k\}$, which is a partition of the integers $\{1,2, \cdots, 4 n\}$, except $j$ and $k$, into $2 n-1$ pairs such that each of the integers $\{1,2, \cdots, m-1, m+1, \cdots, n\}$ appears exactly twice as a difference, while the integer $m$ appears exactly once. These partitions are equivalent to $(p, q)$-extended $m$-near-2-fold Skolem sequences of order $n$. Billington uses these partitions to construct balanced ternary designs. A balanced ternary design, $B T D$, is a pair $(V, B)$, where $V$ is a set of $v$ elements and $B$ is a collection of blocks of size $k$ such that each element in $V$ occurs 0,1 or 2 times in each block. We define the index of the design as

$$
\lambda=\sum_{m=1}^{B} n_{i m} n_{j m},
$$

where $1 \leq i<j \leq v$ and $n_{\text {im }}$ denotes the number of times the element $i$ occurs in block $m$. In particular, she used the pairings of the form $P^{2}(1, m / 2) /(m / 2)-\{m / 2+1,3 m / 2+1\}$ to construct balanced ternary designs with block size three and index two. She used these pairings to construct $m-1$ triples of the form $\left\{x_{i}, y_{i}, z_{i}\right\}$, where $x_{i}+y_{i}=z_{i}, x_{2 i-1}=x_{2 i}=i$ for $1 \leq i \leq m / 2-1$ and $x_{m-1}=m / 2$. She then used these triples to construct blocks of the form $\left\{0, x_{i}, z_{i}\right\}(\bmod 3 m)$, in addition to $\{0,0, m\}$ $(\bmod 3 \mathrm{~m})$, which were base blocks of a $B T D$ of order $V=3 \mathrm{~m}$ and index 2. For example, for $V=12$, the pairing $P^{2}(1,2) / 2-\{3,7\}$ can be used to construct the triples $\{1,2,3\},\{1,5,6\}$, and $\{2,3,5\}$, which gives us base blocks $\{0,0,4\},\{0,1,3\},\{0,1,6\}$ and $\{0,2,5\}(\bmod 12)$. 
Another generalization of Skolem sequences are Langford sequences. Introduced by Langford ,[5], a Langford sequence of order $n$ and defect $d$ is equivalent to a Skolem sequence of order $n$ with the exception that the smallest integer in a Langford sequence is $d$ instead of 1 . For example, the sequence $(3,4,5,3,2,4,2,5)$ is a Langford sequence of order 5 and defect $d=2$. This sequence is also known as a perfect Langford sequence. A hooked Langford sequence of order $n$ and defect $d$ is a Langford sequence with the second last position of the sequence containing a hook. The length of these sequences is $l=n-d+1$.

It was while working with Langford sequences that Priday, [13], introduced the concept of a looped set. Informally, a set $\{d, d+1, \cdots, n\}$ is called a looped Langford set if there exists two Langford sequences of order $n$ and defect $d$, one with a hook two places from the last entry of the sequence and the other with hooks two places and one place from the last entry. In [19], Shalaby and Stuckless refer to these sequences collectively as a looped Langford sequence of order $n$ and defect $d$. For example, given the set $\{2,3,4,5\}$, we can form the sequences $(2,4,2,5,3,4,0,3,5)$ and $(3,5,2,3,2,4,5,0,0,4)$. Linek and Jiang, [7], use a more general result to indirectly show that the necessary conditions for the existence of looped Langford sequences are also sufficient for the case when $d=2$.

Using the sequences introduced by Billington and Priday as motivation, we prove that the necessary conditions are also sufficient for the existence of $(2 n-3)$-extended $m$-near-Skolem sequences (equivalent to $P(1, n) / m-\{2 n-$ $3\})$ and hooked $(2 n-2)$-extended $m$-near-Skolem sequences (equivalent to $P(1, n) / m-\{2 n-2,2 n-1\})$. The following sequences are examples of a 9-extended 4-near-Skolem sequence of order 6 and a hooked 10-extended 4near-Skolem sequence of order 6 , respectively: $(6,3,1,1,3,5,6,2,0,2,5)$ and $(6,2,5,2,1,1,6,5,3,0,0,3)$. We then use these sequences in the construction of other generalizations of Skolem sequences.

\section{Preliminaries}

In this section we state some preliminary definitions and theorems.

Definition 1 A Skolem sequence of order $n$ is a sequence $S_{n}=\left(s_{1}, s_{2}, \cdots, s_{2 n}\right)$ of $2 n$ positive integers such that the following condition hold:

1. for each $k \in\{1,2, \cdots, n\}$ there exists exactly two elements $s_{i}, s_{j} \in S$ such that

$s_{i}=s_{j}=k$

2. if $s_{i}=s_{j}=k$, then $|i-j|=k$ 
Theorem 1 (Skolem, [21]) A Skolem sequence of order $n$ exists if and only if

$n \equiv 0,1(\bmod 4)$.

Definition $2 A$-extended Skolem sequence of order $n$ is a sequence $S_{n}(t)=$ $\left(s_{1}, s_{2}, \cdots, s_{2 n+1}\right)$ of $2 n+1$ non-negative integers which satisfies conditions (1),(2) for Skolem sequences with the added condition $s_{t}=0$ for some $t \in$ $\{1,2, \cdots, 2 n+1\}$. A hooked Skolem sequence of order $n$ is an extended Skolem sequence of order $n$ with $s_{2 n}=0$.

Theorem 2 (Abrham and Kotzig, [1]) An extended Skolem sequence of order $n$ exists for all admissible $n$.

Theorem 3 (Baker [2]) A k-extended Skolem sequence of order $n$ exists if and only if one of the following is true:

1. $n \equiv 0,1(\bmod 4)$ and $k$ is odd

2. $n \equiv 2,3(\bmod 4)$ and $k$ is even

Definition $3 A$ hooked $t$-extended Skolem sequence of order $n$ is a sequence $h S_{n}(t)=\left(s_{1}, s_{2}, \cdots, s_{2 n+2}\right)$ of $2 n+2$ non-negative integers which satisfies conditions (1), (2) for Skolem sequences with the added condition $s_{2 n+1}=s_{t}=$ 0 for some $t \in\{1,2, \cdots, 2 n, 2 n+2\}$.

Theorem 4 (Linek and Jiang [7]) With the exception of $(k, n)=(2,1)$, a hooked $k$-extended Skolem sequence of order $n$ exists if and only if one of the following is true:

1. $n \equiv 0,1(\bmod 4)$ and $k$ is even

2. $n \equiv 2,3(\bmod 4)$ and $k$ is odd

Definition $4 A$ Langford sequence of order $n$ and defect $d, n \geq d$ is a sequence $L_{n}^{d}=\left(l_{1}, l_{2}, \cdots, l_{2(n-d+1)}\right)$ such that the following conditions hold:

1. for each $k \in\{d, d+1, \cdots, n\}$ there exist exactly two elements $l_{i}, l_{j} \in L$ such that

$l_{i}=l_{j}=k$

2. if $l_{i}=l_{j}=k$, then $|i-j|=k$

This sequence is also known as a perfect Langford sequence. The length of the sequence is defined as $l=n-d+1$. 
Theorem 5 (Simpson [20]) A Langford sequence of length $l$ and defect $d$ exists if and only if

1. $l \geq 2 d-1$

2. $l \equiv 0,1(\bmod 4)$ for $d$ odd and $l \equiv 0,3(\bmod 4)$ for $d$ even

Definition $5 A$ hooked Langford sequence of order $n$ and defect $d, n \geq d$ is a sequence $h L_{n}^{d}=\left(l_{1}, l_{2}, \cdots, l_{2(n-d+1)+1}\right)$ which satisfies conditions (1), (2) of a Langford sequence with the added condition $l_{2(n-d+1)}=0$.

Theorem 6 (Simpson [20]) A hooked Langford sequence of length $l$ and defect $d$ exists if and only if

1. $l(l+1-2 d) \geq 0$

2. $l \equiv 2,3(\bmod 4)$ for $d$ odd and $l \equiv 1,2(\bmod 4)$ for $d$ even

Definition 6 A looped Langford set is a pair of sequences $(\mathcal{L}, \mathcal{K})$ of order $n$ and defect $d, n \geq d, \mathcal{L}_{n}=\left(l_{1}, l_{2}, \cdots, l_{2(n-d+1)+1}\right)$ and $\mathcal{K}_{n}=\left(k_{1}, k_{2}, \cdots, k_{2(n-d+1)+2}\right)$ satisfying conditions (1), (2) of a Langford sequence with the added conditions $l_{2(n-d+1)-1}=k_{2(n-d+1)}=k_{2(n-d+1)+1}=0$. If such a set exists, we refer to the two sequences collectively as a looped Langford sequence of order $n$ and defect $d$.

Theorem 7 (Linek and Jiang [7]) A looped Langford sequence of order $n$ and defect $d=2$ exists if and only if $n \equiv 0,1(\bmod 4)$

Definition 7 An $m$-near-Skolem sequence of order $n$ and defect $m$ is a sequence $m-S_{n}=\left(s_{1}, s_{2}, \cdots, s_{2 n-2}\right)$ of $2 n-2$ non-negative integers such that the following conditions hold:

1. for each $k \in\{1,2, \cdots, m-1, m+1, \cdots, n\}$ there exist exactly two elements $s_{i}, s_{j} \in S$ such that $s_{i}=s_{j}=k$

2. if $s_{i}=s_{j}=k$, then $|i-j|=k$

Theorem 8 (Shalaby [16]) An m-near-Skolem sequence of order $n$ exists if and only if one of the following is true:

1. $n \equiv 0,1(\bmod 4)$ and $m$ is odd

2. $n \equiv 2,3(\bmod 4)$ and $m$ is even 
Definition $8 A$-extended $m$-near-Skolem sequence of order $n$ and defect $m$ is a sequence $m-S_{n}(t)=\left(s_{1}, s_{2}, \cdots, s_{2 n-1}\right)$ of $2 n-1$ non-negative integers such that conditions (1), (2) of m-near-Skolem sequences are satisfied, with the added condition $s_{t}=0$, for some $t \in\{1,2, \cdots, 2 n, 2 n-1\}$. A hooked $m$-nearSkolem sequence of order $n$ is an extended $m$-near-Skolem sequence of order $n$ with $s_{2 n-2}=0$.

Although the existence of $t$-extended $m$-near-Skolem sequences is still an open question for general $t$, the case has been solved for $t=2 n-2$ (and $t=2$ ) by Shalaby:

Theorem 9 (Shalaby [16]) A hooked m-near-Skolem sequence of order $n$ exists if and only if one of the following is true:

1. $n \equiv 0,1(\bmod 4)$ and $m$ is even

2. $n \equiv 2,3(\bmod 4)$ and $m$ is odd

Definition $9 A$ hooked $t$-extended $m$-near-Skolem sequence of order $n$ and defect mis a sequence $h m-S_{n}(t)=\left(s_{1}, s_{2}, \cdots, s_{2 n}\right)$ of 2 n non-negative integers which satisfies conditions (1), (2) for m-near-Skolem sequences with the added condition $s_{2 n-1}=s_{t}=0$ for some $t \in\{1,2, \cdots, 2 n-1,2 n\}$.

Throughout the remainder of the article, we will let $\mathcal{O}_{n}^{m}$ and $\mathcal{T}_{n}^{m}$ denote a $(2 n-3)$-extended $m$-near-Skolem sequence of order $n$ and a hooked $(2 n-2)$ extended $m$-near-Skolem sequence of order $n$, respectively.

\section{Necessity}

In this section we find the necessary conditions for the existence of $\mathcal{O}_{n}^{m}$ and $\mathcal{T}_{n}^{m}$. We determine all the possible values $m$ can take given a certain order $n$.

Theorem 10 The sequences $\mathcal{O}_{n}^{m}$ and $\mathcal{T}_{n}^{m}$ exists only if one of the following is true:

1. $n \equiv 0,1(\bmod 4)$ and $m$ is odd

2. $n \equiv 2,3(\bmod 4)$ and $m$ is even

Proof: Consider first the case of $\mathcal{O}_{n}^{m}$. Let $\mathcal{O}_{n}^{m}=\left(s_{1}, s_{2}, \ldots s_{2 n-1}\right)$ be the sequence in question. Let the ordered pairs $(i, j)$ be the subscripts of $s_{i}, s_{j}$ when $s_{i}=s_{j}=k$. Then 
(a)

$$
\sum_{k=1, k \neq m}^{n}(i+j)=\frac{(2 n)(2 n-1)}{2}-(2 n-3)=2 n^{2}-3 n+3
$$

(b)

$$
\sum_{k=1, k \neq m}^{n}(j-i)=\frac{(n)(n+1)}{2}-m
$$

Adding (a) and (b) together gives us

$$
2 \sum_{k=1, k \neq m}^{n} j=\frac{5 n^{2}-5 n-2 m+6}{2}
$$

Since the left hand side of the equation must be an integer, the number $\left(5 n^{2}-\right.$ $5 n-2 m+6$ ) must be divisible by 4 . Solving for $n$ and $m$ we obtain the necessary conditions.

Similarly, for $\mathcal{T}_{n}^{m}$, we have

$\left(a^{\prime}\right)$

$$
\sum_{k=1, k \neq m}^{n}(i+j)=\frac{(2 n)(2 n+1)}{2}-(2 n-1)-(2 n-2)=2 n^{2}-3 n+3
$$

$$
\sum_{k=1, k \neq m}^{n}(j-i)=\frac{(n)(n+1)}{2}-m
$$

Adding $\left(a^{\prime}\right)$ and $\left(b^{\prime}\right)$ together gives us

$$
2 \sum_{k=1, k \neq m}^{n} j=\frac{5 n^{2}-5 n-2 m+6}{2}
$$

Since the left hand side of the equation must be an integer, the number $\left(5 n^{2}-\right.$ $5 n-2 m+6)$ must be divisible by 4 . Solving for $n$ and $m$ we obtain the necessary conditions. 


\section{Sufficiency}

In this section we show that the necessary conditions given in Theorem 10 are also sufficient. The main results are found in Theorems 13,14.

In this section, we make use of a method incorporated in [3], where the authors note that two hooked sequences can be hooked together to form a sequence with no hooks. This is done by "hooking" the first sequence together with the reverse of the second. The last entry in the first sequence replaces the hook in the reverse of the second and the first entry in the reverse of the second replaces the hook in the first. For example, $(3,1,1,3,2,0,2)$ and $(1,1,2,0,2)$ can be hooked together to give $(3,1,1,3,2,2,2,2,1,1)$.

Theorem 11 If $n \equiv 2,7(\bmod 8)$, then $\mathcal{O}_{n}^{m}$ and $\mathcal{T}_{n}^{m}$ exists. The case $n=2$ is an exception.

\section{Proof:}

Let $n=8 s+2$. For $s=1$ we have the sequences

$\mathcal{T}_{10}^{4}=(8,6,2,9,2,5,7,6,8,10,5,3,9,7,3,1,1,0,0,10)$ and

$\mathcal{O}_{10}^{4}=(3,10,8,3,5,1,1,7,9,5,8,10,6,2,7,2,0,9,6)$. So assume $s \geq 2$. Вy theorem 6 , we can construct a hooked Langford sequence of order $n$ and defect $d=5$. We can then hook this sequence together with the sequences $(2,0,2,1,1,3,0,0,3)$ and $(3,0,2,3,2,0,1,1)$ to form $\mathcal{T}_{n}^{m}$ and $\mathcal{O}_{n}^{m}$, respectively. Similarly, we can construct $\mathcal{T}_{n}^{m}$ and $\mathcal{O}_{n}^{m}$ for $n=8 s+7, s \geq 1$. For $n=7$, we have the sequences $(6,7,3,1,1,3,6,5,7,2,0,2,5)$ and

$(2,7,2,1,1,5,3,6,7,3,5,0,0,6)$.

Theorem 12 If $n=8 s$, then $\mathcal{O}_{n}^{m}$ and $\mathcal{T}_{n}^{m}$ exists, where $m=2 t-1$ for all $1 \leq t \leq s$.

Proof: Let $n=8 s$, where $s \geq 1$. For $s=t=m=1$ we can construct a looped Langford sequence of order $n$ and defect $d=2$. So we can assume $s \geq t \geq 2$. We distinguish between two separate cases:

\section{Case $1: t \equiv 0(\bmod 2)$}

We first construct a Langford sequence, $L_{n}^{2 t+1}=\left(l_{1}, l_{2}, \cdots, l_{2(n-2 t)}\right)$, of order $n$ and $d=2 t+1$. We next construct a hooked $(2 t-1)$-extended Skolem sequence, $h S_{2 t-2}(2 t-1)=\left(s_{1}, s_{2}, \cdots, s_{4 t-2}\right)$, of order $n^{\prime}=2 t-2$. We then place the difference $i=2 t$ in position $s_{2 t-1}$ of $h S_{2 t-2}(2 t-1)$ and also at the end . Appending this newly constructed sequence to the end of $L_{n}^{2 t+1}$ gives us $\mathcal{O}_{n}^{m}$.

Next we construct a $2 t$-extended Skolem sequence $S_{2 t-2}(2 t)=\left(e_{1}, e_{2}, \cdots, e_{4 t-3}\right)$, of order $n^{\prime}=2 t-2$. We then replace the hook in this sequence with the difference $i=2 t$ and append $0,0,2 t$ to the end. Appending this newly constructed sequence to the end of $L_{n}^{2 t+1}$ gives us $\mathcal{T}_{n}^{m}$. 


\section{Case 2: $t \equiv 1(\bmod 2)$}

We first construct a Langford sequence, $L_{n}^{2 t}=\left(l_{1}, l_{2}, \cdots, l_{2(n-2 t+1)}\right)$, of order $n$ and $d=2 t$. We next construct a hooked $(4 t-4)$-extended Skolem sequence, $h S_{2 t-2}(4 t-4)=\left(s_{1}, s_{2}, \cdots, s_{4 t-2}\right)$, of order $n^{\prime}=2 t-2$. Appending $h S_{2 t-2}(4 t-4)$ to the end of $L_{n}^{2 t}$ gives us $\mathcal{T}_{n}^{m}$.

Next we construct $(4 t-5)$-extended Skolem sequence $S_{4 t-3}(4 t-5)=$ $\left(e_{1}, e_{2}, \cdots, e_{4 t-3}\right)$, of order $n^{\prime}=2 t-2$. Appending $S_{4 t-3}(4 t-5)$ to the end of $L_{n}^{2 t}$ gives us $\mathcal{O}_{n}^{m}$.

Lemma 1 For all admissible $n$, the sequences $\mathcal{O}_{n}^{m}$ and $\mathcal{T}_{n}^{m}$ exists, where $m=1$ or $n$.

Proof: We first note that the sequence $\mathcal{O}_{n}^{m}$ does not exist for $n=1$ and $(n, m)=(3,2)$, and the sequence $\mathcal{T}_{n}^{m}$ does not exist for $n=1$ and $n=2$. For $n \equiv 0,1(\bmod 4), n \geq 4$ and $m=1$, we have a looped Langford sequence of order $n$ and defect $d=2$. For $n \equiv 1,2(\bmod 4), n \geq 5$, and $m=n$, we have a Langford sequence of order $n-1$ and defect $d=2$ with 1,1 appended to the end.

Theorem $13 A(2 n-3)$-extended m-near-Skolem sequence of order $n$ and defect $m$ exists if and only if one of the following is true:

1. $n \equiv 0,1(\bmod 4)$ and $m$ is odd

2. $n \equiv 2,3(\bmod 4)$ and $m$ is even

The cases $n=1$ and $(n, m)=(3,2)$ are exceptions.

Proof: Necessity was shown in Theorem 10. For sufficiency we first look at some examples with small $m$. For $n \equiv 0,1(\bmod 4)$ and $m=1$, see Lemma 1. For $n \equiv 1,2(\bmod 4)$ and $m=n$, see Lemma 1 . For $n \equiv 2,7(\bmod 8)$ and $m=4$, see Theorem 11 . For $n \equiv 2,3(\bmod 4), n \geq 7$ and $m=2$, we have a Langford sequence of order $n$ and $d=3$ with $0,1,1$ appended to the end. For the remaining $n$ and $m$ we distinguish eight cases. In each case the solution is given in the form of a table, where the columns $i, j$ denote the first and second appearance, respectively, of the difference $k$.

Case $1: n \equiv 0(\bmod 8)$.

Let $n=8 s, m=2 t+1$. For $m \leq 2 s-1$ see Theorem 12. For $n>m>2 s-1$ and $n>8$, the solution is given by the following table (ignore the lines ${ }^{*}$ when 
$s=2)$.

\begin{tabular}{|l|l|l|l|}
\hline$i$ & $j$ & $k$ & \\
\hline $1+r$ & $8 s-r$ & $8 s-2 r-1$ & $0 \leq r \leq 4 s-t-2$ \\
$4 s-t+r$ & $4 s+t-r-1$ & $2 t-2 r-1$ & $0 \leq r \leq t-s-1$ \\
$4 s+t$ & $4 s+t+1$ & 1 & $\cdots \cdots$ \\
$3 s+r$ & $11 s-r$ & $8 s-2 r$ & $0 \leq r \leq 2 s-1$ \\
$8 s+r+1$ & $12 s-r+1$ & $4 s-2 r$ & $0 \leq r \leq s-1$ \\
$11 s+1$ & $13 s$ & $2 s-1$ & $\cdots \cdots$ \\
$14 s-1$ & $16 s-1$ & $2 s$ & $\cdots \cdots$ \\
$12 s+r+2$ & $14 s-r-2$ & $2 s-4-2 r$ & $0 \leq r \leq s-4$ \\
$* 13 s-1$ & $15 s-3$ & $2 s-2$ & $\cdots \cdots$ \\
$* 13 s+1$ & $15 s-2$ & $2 s-3$ & $\cdots \cdots$ \\
$14 s+r$ & $16 s-r-5$ & $2 s-5-r$ & $0 \leq r \leq s-4$ \\
$16 s-4$ & $16 s-2$ & 2 & $\cdots \cdots$ \\
\hline
\end{tabular}

To complete the proof we list below the $(2 n-3)$-extended $m$-near-Skolem sequences of order 8 and all required defects:

For $n=8$ and $m=3,5,7$ :

$$
\begin{aligned}
& 7,5,6,1,1,8,5,7,6,2,4,2,0,8,4 \\
& 7,3,6,2,3,2,8,7,6,4,1,1,0,4,8 \\
& 5,3,8,6,3,5,2,4,2,6,8,4,0,1,1
\end{aligned}
$$

Case $2: n \equiv 1(\bmod 8)$.

Let $n=8 s+1, m=2 t+1$. For $n>m>1$, the solution is given by the following table (ignore the line ${ }^{*}$ when $s=1$ ).

\begin{tabular}{|l|l|l|l|}
\hline$i$ & $j$ & $k$ & \\
\hline $1+r$ & $8 s-r+2$ & $8 s-2 r+1$ & $0 \leq r \leq 4 s-t-1$ \\
$4 s-t+r+1$ & $4 s+t-r$ & $2 t-2 r-1$ & $0 \leq r \leq t-2$ \\
$4 s+t+1$ & $4 s+t+2$ & 1 & $\cdots \cdots$ \\
$4 s+r$ & $12 s-r$ & $8 s-2 r$ & $0 \leq r \leq 1$ \\
$8 s+2 r+4$ & $16 s-2 r$ & $8 s-4 r-4$ & $0 \leq r \leq s-2$ \\
$* 8 s+2 r+3$ & $16 s-2 r-3$ & $8 s-4 r-6$ & $0 \leq r \leq s-1$ \\
$10 s+r+2$ & $14 s-r-2$ & $4 s-2 r-4$ & $0 \leq r \leq 2 s-4$ \\
$12 s+1$ & $16 s+1$ & $4 s$ & $\cdots \ldots$ \\
$14 s$ & $14 s+2$ & 2 & $\ldots \ldots$ \\
\hline
\end{tabular}

Case $3: n \equiv 2(\bmod 8)$.

Let $n=8 s+2, m=2 t$. This is true for all subsequent cases and so we will omit to mention it. For $n \geq m>4$ and $n>10$, the solution is given by the following table (ignore the line ${ }^{*}$ when $s=2$ ). 


\begin{tabular}{|l|l|l|l|}
\hline$i$ & $j$ & $k$ & \\
\hline $1+r$ & $8 s-r+3$ & $8 s-2 r+2$ & $0 \leq r \leq 4 s-t$ \\
$4 s-t+r+2$ & $4 s+t-r$ & $2 t-2 r-2$ & $0 \leq r \leq t-4$ \\
$4 s+t+1$ & $4 s+t+2$ & 1 & $\ldots \ldots$ \\
$4 s+r-1$ & $12 s-r$ & $8 s-2 r+1$ & $0 \leq r \leq 4$ \\
$8 s+2 r+5$ & $16 s-2 r-4$ & $8 s-4 r-9$ & $0 \leq r \leq s-3$ \\
$* 8 s+2 r+4$ & $16 s-2 r-7$ & $8 s-4 r-11$ & $0 \leq r \leq s-2$ \\
$10 s+r+1$ & $14 s-r-2$ & $4 s-2 r-3$ & $0 \leq r \leq 2 s-5$ \\
$12 s+1$ & $16 s$ & $4 s-1$ & $\ldots \ldots$ \\
$16 s-3$ & $16 s+2$ & 5 & $\ldots \ldots$ \\
$16 s-1$ & $16 s+3$ & 4 & $\ldots \ldots$ \\
$16 s-5$ & $16 s-2$ & 3 & $\ldots \ldots$ \\
$14 s-2$ & $14 s$ & 2 & \\
\hline
\end{tabular}

To complete the proof we need complete listings for $n=10$ and $n=2$.

For $n=2: 0,1,1$

For $n=10$ and $m=6,8$ :

$$
\begin{aligned}
& 3,10,6,3,1,1,4,7,6,9,4,10,5,2,7,2,0,5,9 \\
& 3,10,8,3,9,1,1,2,7,2,8,10,5,9,4,7,0,5,4
\end{aligned}
$$

Case $4: n \equiv 3(\bmod 8)$.

Let $n=8 s+3, m=2 t$. For $n \geq m>2$ and $n>11$, the solution is given by the following table.

\begin{tabular}{|l|l|l|l|}
\hline$i$ & $j$ & $k$ & \\
\hline $1+r$ & $8 s-r+3$ & $8 s-2 r+2$ & $0 \leq r \leq 4 s-t$ \\
$4 s-t+r+2$ & $4 s+t-r$ & $2 t-2 r-2$ & $0 \leq r \leq t-3$ \\
$4 s+t+1$ & $4 s+t+2$ & 1 & $\cdots \cdots$ \\
$4 s+r$ & $12 s-r+3$ & $8 s-2 r+3$ & $0 \leq r \leq 2$ \\
$8 s+2 r+5$ & $16 s-2 r+2$ & $8 s-4 r-3$ & $0 \leq r \leq s-3$ \\
$8 s+2 r+4$ & $16 s-2 r-1$ & $8 s-4 r-5$ & $0 \leq r \leq s-2$ \\
$10 s+r+1$ & $14 s-r+2$ & $4 s-2 r+1$ & $0 \leq r \leq 2 s-2$ \\
$12 s$ & $16 s+5$ & $4 s+5$ & $\cdots \cdots$ \\
$16 s+1$ & $16 s+4$ & 3 & $\cdots$ \\
$14 s+4$ & $14 s+6$ & 2 & $\cdots$ \\
\hline
\end{tabular}

To complete the proof we list below $(2 n-3)$-extended $m$-near-Skolem sequences of order 11 and all required defects:

For $n=11$ and $m=4,6,8,10$ 
$3,7,10,3,1,1,8,6,7,11,9,5,10,6,8,2,5,2,0,9,11$

$11,9,7,5,3,10,8,3,5,7,9,11,1,1,8,10,4,2,0,2,4$

$11,9,7,5,3,10,6,3,5,7,9,11,6,1,1,10,4,2,0,2,4$

$11,9,7,5,3,8,6,3,5,7,9,11,6,8,1,1,4,2,0,2,4$

Case $5: n \equiv 4(\bmod 8)$.

Let $n=8 s+4, m=2 t+1$. For $n \geq m>1$ and $n>4$, the solution is given by the following table.

\begin{tabular}{|l|l|l|l|}
\hline$i$ & $j$ & $k$ & \\
\hline $1+r$ & $8 s-r+4$ & $8 s-2 r+3$ & $0 \leq r \leq 4 s-t$ \\
$4 s-t+r+2$ & $4 s+t-r+1$ & $2 t-2 r-1$ & $0 \leq r \leq t-2$ \\
$4 s+t+2$ & $4 s+t+3$ & 1 & $\cdots \cdots$ \\
$4 s+r+1$ & $12 s-r+5$ & $8 s-2 r+4$ & $0 \leq r \leq 1$ \\
$8 s+2 r+6$ & $16 s-2 r+6$ & $8 s-4 r$ & $0 \leq r \leq s-2$ \\
$8 s+2 r+5$ & $16 s-2 r+3$ & $8 s-4 r-2$ & $0 \leq r \leq s-1$ \\
$10 s+r+4$ & $14 s-r+4$ & $4 s-2 r$ & $0 \leq r \leq 2 s-2$ \\
$12 s+3$ & $16 s+7$ & $4 s+4$ & $\cdots \cdots$ \\
$14 s+6$ & $14 s+8$ & 2 & $\ldots \ldots$ \\
\hline
\end{tabular}

To complete the proof we list below a $(2 n-3)$-extended 3-near-Skolem sequences of order 4:

$$
1,1,4,2,0,2,4
$$

Case $6: n \equiv 5(\bmod 8)$.

Let $n=8 s+5, m=2 t+1$. For $n \geq 13$ and $m=3$ we have a Langford sequence with $d=5$ and $1,1,4,2,0,2,4$ appended to the end. For $n \geq 16$ and $m=5$ we have a Langford sequence with $d=6$ and $3,1,1,3,4,2,0,2,4$ appended to the end. For $n \geq m>5$ and $n>13$, the solution is given by the following table (ignore the line ${ }^{*}$ when $s=2$ ). 


\begin{tabular}{|l|l|l|l|}
\hline$i$ & $j$ & $k$ & \\
\hline $1+r$ & $8 s-r+6$ & $8 s-2 r+5$ & $0 \leq r \leq 4 s-t+1$ \\
$4 s-t+r+3$ & $4 s+t-r+2$ & $2 t-2 r-1$ & $0 \leq r \leq t-4$ \\
$4 s+t+3$ & $4 s+t+4$ & 1 & $\cdots \cdots$ \\
$4 s+r$ & $12 s-r+4$ & $8 s-2 r+4$ & $0 \leq r \leq 5$ \\
$8 s+2 r+8$ & $16 s-2 r$ & $8 s-4 r-8$ & $0 \leq r \leq s-3$ \\
$* 8 s+2 r+7$ & $16 s-2 r-3$ & $8 s-4 r-10$ & $0 \leq r \leq s-2$ \\
$10 s+r+4$ & $14 s-r$ & $4 s-2 r-4$ & $0 \leq r \leq 2 s-6$ \\
$12 s+5$ & $16 s+5$ & $4 s$ & $\cdots \ldots$ \\
$16 s+3$ & $16 s+9$ & 6 & $\ldots \ldots$ \\
$16 s+1$ & $16 s+6$ & 5 & $\cdots$ \\
$16 s+4$ & $16 s+8$ & 4 & $\cdots$ \\
$16 s-1$ & $16 s+2$ & 3 & $\cdots$ \\
$14 s+2$ & $14 s+4$ & 2 & $\ldots$ \\
\hline
\end{tabular}

To complete the proof we need complete listings for $n=5,13$ : For $n=5$ and $m=3$ :

$$
4,1,1,5,4,2,0,2,5
$$

For $n=13$ and $m=5,7,9,11$ :

$$
\begin{aligned}
& 12,10,8,6,13,3,9,11,3,6,8,10,12,1,1,9,7,13,11,2,4,2,0,7,4 \\
& 12,10,8,6,13,3,9,11,3,6,8,10,12,1,1,9,4,13,11,5,4,2,0,2,5 \\
& 12,10,8,6,13,3,7,11,3,6,8,10,12,7,5,1,1,13,11,5,4,2,0,2,4 \\
& 12,10,8,6,13,3,7,9,3,6,8,10,12,7,4,5,9,13,4,2,5,2,0,1,1
\end{aligned}
$$

Case $7: n \equiv 6(\bmod 8)$.

Let $n=8 s+6, m=2 t$. For $n \geq m>2$ and $n>6$, the solution is given by the following table.

\begin{tabular}{|l|l|l|l|}
\hline$i$ & $j$ & $k$ & \\
\hline $1+r$ & $8 s-r+7$ & $8 s-2 r+6$ & $0 \leq r \leq 4 s-t+2$ \\
$4 s-t+r+4$ & $4 s+t-r+2$ & $2 t-2 r-2$ & $0 \leq r \leq t-3$ \\
$4 s+t+3$ & $4 s+t+4$ & 1 & $\ldots \ldots$ \\
$4 s+r+2$ & $12 s-r+7$ & $8 s-2 r+5$ & $0 \leq r \leq 2$ \\
$8 s+2 r+9$ & $16 s-2 r+8$ & $8 s-4 r-1$ & $0 \leq r \leq s-2$ \\
$8 s+2 r+8$ & $16 s-2 r+5$ & $8 s-4 r-3$ & $0 \leq r \leq s-1$ \\
$10 s+r+7$ & $14 s-r+6$ & $4 s-2 r-1$ & $0 \leq r \leq 2 s-3$ \\
$12 s+8$ & $16 s+11$ & $4 s+3$ & $\ldots \ldots$ \\
$16 s+7$ & $16 s+10$ & 3 & $\ldots \ldots$ \\
$14 s+8$ & $14 s+10$ & 2 & $\ldots \ldots$ \\
\hline
\end{tabular}


To complete the proof we list below $(2 n-3)$-extended $m$-near-Skolem sequences of order 6 and all required defects:

For $n=6$ and $m=2,4$ :

$$
\begin{aligned}
& 6,4,1,1,5,4,6,3,0,5,3 \\
& 6,3,1,1,3,5,6,2,0,2,5
\end{aligned}
$$

Case $8: n \equiv 7(\bmod 8)$.

Let $n=8 s+7, m=2 t$. For $n \geq m>4$ and $n>7$, the solution is given by the following table.

\begin{tabular}{|l|l|l|l|}
\hline$i$ & $j$ & $k$ & \\
\hline $1+r$ & $8 s-r+7$ & $8 s-2 r+6$ & $0 \leq r \leq 4 s-t+2$ \\
$4 s-t+r+4$ & $4 s+t-r+2$ & $2 t-2 r-2$ & $0 \leq r \leq t-4$ \\
$4 s+t+3$ & $4 s+t+4$ & 1 & $\cdots \cdots$ \\
$4 s+r+1$ & $12 s-r+8$ & $8 s-2 r+7$ & $0 \leq r \leq 4$ \\
$8 s+2 r+9$ & $16 s-2 r+6$ & $8 s-4 r-3$ & $0 \leq r \leq s-3$ \\
$8 s+2 r+8$ & $16 s-2 r+3$ & $8 s-4 r-5$ & $0 \leq r \leq s-2$ \\
$10 s+r+5$ & $14 s-r+6$ & $4 s-2 r+1$ & $0 \leq r \leq 2 s-3$ \\
$12 s+3$ & $16 s+8$ & $4 s+5$ & $\cdots \cdots$ \\
$16 s+7$ & $16 s+12$ & 5 & $\ldots \ldots$ \\
$16 s+5$ & $16 s+9$ & 4 & $\ldots \ldots$ \\
$16 s+10$ & $16 s+13$ & 3 & $\ldots \ldots$ \\
$14 s+8$ & $14 s+10$ & 2 & $\ldots \ldots$ \\
\hline
\end{tabular}

To complete the proof we list below $(2 n-3)$-extended 6-near-Skolem sequence of order 7 :

$$
7,5,3,1,1,3,5,7,4,2,0,2,4
$$

Theorem 14 A hooked $(2 n-2)$-extended $m$-near-Skolem sequence of order $n$ and defect $m$ exists if and only if one of the following is true:

1. $n \equiv 0,1(\bmod 4)$ and $m$ is odd

2. $n \equiv 2,3(\bmod 4)$ and $m$ is even

The cases $n=1$ or 2 are exceptions.

Proof: Necessity was shown in Theorem 10. For sufficiency we first look at some examples with small $m$. For $n \equiv 0,1(\bmod 4)$ and $m=1$, see Lemma 1. For $n \equiv 1,2(\bmod 4)$ and $m=n$, see Lemma 1 . For $n \equiv 2,7(\bmod 8)$ and 
$m=4$, see Theorem 11 . For $n \equiv 2,3(\bmod 4), n \geq 10$ and $m=2$, we have a Langford sequence of order $n$ and $d=4$ with $1,1,3,0,0,3$ appended to the end. For the remaining $n$ and $m$ we distinguish eight cases.For sufficiency we distinguish eight cases. In each case the solution is given in the form of a table, where the columns $i, j$ denote the first and second appearance, respectively, of the difference $k$.

Case $1: n \equiv 0(\bmod 8)$.

Let $n=8 s, m=2 t+1$. For $m \leq 2 s-1$ see Theorem 12. For $n>m>2 s-1$ and $n>8$, the solution is given by the following table.

\begin{tabular}{|l|l|l|l|}
\hline$i$ & $j$ & $k$ & \\
\hline $1+r$ & $8 s-r$ & $8 s-2 r-1$ & $0 \leq r \leq 4 s-t-2$ \\
$4 s-t+r$ & $4 s+t-r-1$ & $2 t-2 r-1$ & $0 \leq r \leq t-s-1$ \\
$4 s+t$ & $4 s+t+1$ & 1 & $\cdots \cdots$ \\
$3 s+r$ & $11 s-r$ & $8 s-2 r$ & $0 \leq r \leq 2 s-1$ \\
$8 s+r+1$ & $12 s-r+1$ & $4 s-2 r$ & $0 \leq r \leq s-1$ \\
$11 s+1$ & $13 s$ & $2 s-1$ & $\cdots \cdots$ \\
$12 s+r+2$ & $14 s-r-1$ & $2 s-2 r-3$ & $0 \leq r \leq s-3$ \\
$13 s+1$ & $15 s-1$ & $2 s-2$ & $\cdots \cdots$ \\
$14 s$ & $16 s$ & $2 s$ & $\cdots \cdots$ \\
$14 s+r+1$ & $16 s-r-3$ & $2 s-2 r-4$ & $0 \leq r \leq s-3$ \\
\hline
\end{tabular}

To complete the proof we list below the hooked $(2 n-2)$-extended nearSkolem sequences of order 8 and all required defects:

For $n=8$ and $m=3,5,7$ :

$6,1,1,4,8,5,6,4,7,2,5,2,8,0,0,7$
$6,4,1,1,8,4,6,3,7,2,3,2,8,0,0,7$
$6,1,1,4,8,3,6,4,3,2,5,2,8,0,0,5$

Case $2: n \equiv 1(\bmod 8)$.

Let $n=8 s+1, m=2 t+1$. For $n>m>1$, the solution is given by the following table. 


\begin{tabular}{|l|l|l|l|}
\hline$i$ & $j$ & $k$ & \\
\hline $1+r$ & $8 s-r+2$ & $8 s-2 r+1$ & $0 \leq r \leq 4 s-t-1$ \\
$4 s-t+r+1$ & $4 s+t-r$ & $2 t-2 r-1$ & $0 \leq r \leq t-2$ \\
$4 s+t+1$ & $4 s+t+2$ & 1 & $\cdots \cdots$ \\
$4 s+r$ & $12 s-r$ & $8 s-2 r$ & $0 \leq r \leq 1$ \\
$8 s+r+3$ & $16 s-r-1$ & $8 s-2 r-4$ & $0 \leq r \leq 2 s-3$ \\
$12 s+2$ & $16 s+2$ & $4 s$ & $\cdots \cdots$ \\
$10 s+2 r+2$ & $14 s-2 r$ & $4 s-2-4 r$ & $0 \leq r \leq s-2$ \\
$10 s+2 r+1$ & $14 s-2 r-3$ & $4 s-4-4 r$ & $0 \leq r \leq s-2$ \\
$14 s-1$ & $14 s+1$ & 2 & $\cdots \cdots$ \\
\hline
\end{tabular}

Case $3: n \equiv 2(\bmod 8)$.

Let $n=8 s+2, m=2 t$. For $n>m>4$ and $n>10$, the solution is given by the following table (ignore the line ${ }^{*}$ when $s=2$ ).

\begin{tabular}{|l|l|l|l|}
\hline$i$ & $j$ & $k$ & \\
\hline $1+r$ & $8 s-r+3$ & $8 s-2 r+2$ & $0 \leq r \leq 4 s-t$ \\
$4 s-t+r+2$ & $4 s+t-r$ & $2 t-2 r-2$ & $0 \leq r \leq t-4$ \\
$4 s+t+1$ & $4 s+t+2$ & 1 & $\ldots \ldots$ \\
$4 s+r-1$ & $12 s-r$ & $8 s-2 r+1$ & $0 \leq r \leq 4$ \\
$8 s+2 r+5$ & $16 s-2 r-4$ & $8 s-4 r-9$ & $0 \leq r \leq s-3$ \\
$* 8 s+2 r+4$ & $16 s-2 r-7$ & $8 s-4 r-11$ & $0 \leq r \leq s-2$ \\
$12 s+1$ & $16 s$ & $4 s-1$ & $\ldots \ldots$ \\
$10 s+r+1$ & $14 s-r-4$ & $4 s-2 r-5$ & $0 \leq r \leq 2 s-6$ \\
$16 s-1$ & $16 s+4$ & 5 & $\cdots \cdots$ \\
$16 s-3$ & $16 s+1$ & 4 & $\ldots \ldots$ \\
$16 s-5$ & $16 s-2$ & 3 & $\ldots$ \\
$14 s-2$ & $14 s$ & 2 & $\ldots$ \\
\hline
\end{tabular}

To complete the proof we list below the hooked $(2 n-2)$-extended $m$-nearSkolem sequences of order $n=10$ and all required defects $m$ :

For $n=10$ and $m=6,8$ :

$8,4,2,9,2,4,5,7,8,10,3,5,9,3,7,1,1,0,0,10$

$6,4,2,9,2,4,6,3,7,10,3,5,9,1,1,7,5,0,0,10$

Case $4: n \equiv 3(\bmod 8)$.

Let $n=8 s+3, m=2 t$. For $n \geq m>2$ and $n>3$, the solution is given by the following table (ignore the line ${ }^{*}$ when $s=1$ ). 


\begin{tabular}{|l|l|l|l|}
\hline$i$ & $j$ & $k$ & \\
\hline $1+r$ & $8 s-r+3$ & $8 s-2 r+2$ & $0 \leq r \leq 4 s-t$ \\
$4 s-t+r+2$ & $4 s+t-r$ & $2 t-2 r-2$ & $0 \leq r \leq t-3$ \\
$4 s+t+1$ & $4 s+t+2$ & 1 & $\cdots \cdots$ \\
$4 s+r$ & $12 s-r+3$ & $8 s-2 r+3$ & $0 \leq r \leq 2$ \\
$8 s+2 r+5$ & $16 s-2 r+2$ & $8 s-4 r-3$ & $0 \leq r \leq s-2$ \\
$* 8 s+2 r+4$ & $16 s-2 r-1$ & $8 s-5-4 r$ & $0 \leq r \leq s-1$ \\
$10 s+r+3$ & $14 s-r$ & $4 s-3-2 r$ & $0 \leq r \leq 2 s-4$ \\
$12 s$ & $16 s+1$ & $4 s+1$ & $\cdots \cdots$ \\
$16 s+3$ & $16 s+6$ & 3 & $\cdots$ \\
$14 s+2$ & $14 s+4$ & 2 & $\cdots$ \\
\hline
\end{tabular}

To complete the proof we list the sequence for $n=3, m=2: 1,1,3,0,0,3$

Case $5: n \equiv 4(\bmod 8)$.

Let $n=8 s+4, m=2 t+1$. For $n \geq 11, m=3$, we have a Langford sequence of order $n$ and defect $d=5$ with $1,1,2,4,2,0,0,4$ appended to the end. For $n \geq m>3$ and $n>4$, the solution is given by the following table (ignore the lines $*$ when $s=1)$.

\begin{tabular}{|l|l|l|l|}
\hline$i$ & $j$ & $k$ & \\
\hline $1+r$ & $8 s-r+4$ & $8 s-2 r+3$ & $0 \leq r \leq 4 s-t$ \\
$4 s-t+r+2$ & $4 s+t-r+1$ & $2 t-2 r-1$ & $0 \leq r \leq t-3$ \\
$4 s+t+2$ & $4 s+t+3$ & 1 & $\cdots \cdots$ \\
$4 s+r$ & $12 s-r+4$ & $8 s-2 r+4$ & $0 \leq r \leq 3$ \\
$* 8 s+2 r+6$ & $16 s-2 r$ & $8 s-4 r-6$ & $0 \leq r \leq s-1$ \\
$8 s+2 r+7$ & $16 s-2 r+3$ & $8 s-4 r-4$ & $0 \leq r \leq s-2$ \\
$10 s+r+5$ & $14 s-r+1$ & $4 s-2 r-4$ & $0 \leq r \leq 2 s-5$ \\
$* 8 s+5$ & $12 s+5$ & $4 s$ & $\cdots \cdots$ \\
$16 s+4$ & $16 s+8$ & 4 & $\ldots \ldots$ \\
$16 s+2$ & $16 s+5$ & 3 & $\ldots$ \\
$14 s+3$ & $14 s+5$ & 2 & $\ldots$ \\
\hline
\end{tabular}

To complete the proof we list the sequence for $n=4, m=3: 1,1,2,4,2,0,0,4$.

Case $6: n \equiv 5(\bmod 8)$.

Let $n=8 s+5, m=2 t+1$. For $n \geq m>1$ and $n>13$, the solution is given by the following table. 


\begin{tabular}{|l|l|l|l|}
\hline$i$ & $j$ & $k$ & \\
\hline $1+r$ & $8 s-r+4$ & $8 s-2 r+3$ & $0 \leq r \leq 4 s-t$ \\
$4 s-t+r+2$ & $4 s+t-r+1$ & $2 t-2 r-1$ & $0 \leq r \leq t-2$ \\
$4 s+t+2$ & $4 s+t+3$ & 1 & $\ldots \ldots$ \\
$4 s+r+1$ & $12 s-r+5$ & $8 s-2 r+4$ & $0 \leq r \leq 1$ \\
$8 s+6$ & $12 s+6$ & $4 s$ & $\ldots \ldots$ \\
$8 s+5$ & $16 s+10$ & $8 s+5$ & $\ldots \ldots$ \\
$10 s+7$ & $12 s+7$ & $2 s$ & $\ldots \ldots$ \\
$8 s+r+7$ & $16 s-r+7$ & $8 s-2 r$ & $0 \leq r \leq 2 s-1$ \\
$12 s+3$ & $14 s+7$ & $2 s+4$ & $\cdots \cdots$ \\
$10 s+r+8$ & $14 s-r+6$ & $4 s-2 r-2$ & $0 \leq r \leq s-4$ \\
$11 s+5$ & $13 s+7$ & $2 s+2$ & $\cdots$ \\
$11 s+r+6$ & $13 s-r+4$ & $2 s-2-2 r$ & $0 \leq r \leq s-4$ \\
$13 s+5$ & $13 s+9$ & 4 & $\cdots \cdots$ \\
$13 s+6$ & $13 s+8$ & 2 & $\cdots$ \\
\hline
\end{tabular}

To complete the proof we need complete listings for $n=5,13$ :

For $n=5$ and $m=3$ :

$1,1,4,2,5,2,4,0,0,5$

For $n=13$ and $m=3,5,7,9,11$ :

$11,9,7,5,12,10,1,1,5,7,9,11,13,6,8,10,12,4,2,6,2,4,8,0,0,13$

By shifting 1, 1 one position to the right and replacing the pair $(2 i+1,2 i+1)$ by $(2 i+3,2 i+3)$ we get all required defects $m$.

Case $7: n \equiv 6(\bmod 8)$.

Let $n=8 s+6, m=2 t$. For $n \geq m>2$ and $n>14$, the solution is given by the following table. 


\begin{tabular}{|l|l|l|l|}
\hline$i$ & $j$ & $k$ & \\
\hline $1+r$ & $8 s-r+7$ & $8 s-2 r+6$ & $0 \leq r \leq 4 s-t+2$ \\
$4 s-t+r+4$ & $4 s+t-r+2$ & $2 t-2 r-2$ & $0 \leq r \leq t-3$ \\
$4 s+t+3$ & $4 s+t+4$ & 1 & $\ldots \ldots$ \\
$4 s+r+2$ & $12 s-r+7$ & $8 s-2 r+5$ & $0 \leq r \leq 2$ \\
$8 s+2 r+8$ & $16 s-2 r+5$ & $8 s-4 r-3$ & $0 \leq r \leq s$ \\
$8 s+2 r+9$ & $16 s-2 r+8$ & $8 s-4 r-1$ & $0 \leq r \leq s-1$ \\
$10 s+r+9$ & $14 s-r+4$ & $4 s-2 r-5$ & $0 \leq r \leq 2 s-5$ \\
$12 s+8$ & $16 s+7$ & $4 s-1$ & $\ldots \ldots$ \\
$16 s+9$ & $16 s+12$ & 3 & $\ldots \ldots$ \\
$14 s+6$ & $14 s+8$ & 2 & $\ldots \ldots$ \\
\hline
\end{tabular}

To complete the proof we need complete listings for $n=6,14$ :

For $n=6$ and $m=2,4$ :

$$
\begin{aligned}
& 6,4,5,1,1,4,6,5,3,0,0,3 \\
& 6,2,5,2,1,1,6,5,3,0,0,3
\end{aligned}
$$

For $n=14$ and $m=4,6,8,10,12$ :

$$
14,12,10,8,6,1,1,13,11,9,6,8,10,12,14,2,7,2,9,11,13,3,5,7,3,0,0,5
$$

By shifting the 1,1 one position to the left and replacing the pair $(2 i, 2 i)$ by the $(2 i+2,2 i+2)$ we get all required defects $m$.

Case 8: $n \equiv 7(\bmod 8)$.

Let $n=8 s+7, m=2 t$. For $n>m>4$ and $n>7$, the solution is given by the following table (ignore the line ${ }^{*}$ when $s=1$ ).

\begin{tabular}{|l|l|l|l|}
\hline$i$ & $j$ & $k$ & \\
\hline $1+r$ & $8 s-r+7$ & $8 s-2 r+6$ & $0 \leq r \leq 4 s-t+2$ \\
$4 s-t+r+4$ & $4 s+t-r+2$ & $2 t-2 r-2$ & $0 \leq r \leq t-4$ \\
$4 s+t+3$ & $4 s+t+4$ & 1 & $\cdots \cdots$ \\
$4 s+r+1$ & $12 s-r+8$ & $8 s-2 r+7$ & $0 \leq r \leq 4$ \\
$8 s+2 r+10$ & $16 s-2 r+7$ & $8 s-4 r-3$ & $0 \leq r \leq s-2$ \\
$* 8 s+2 r+9$ & $16 s-2 r+4$ & $8 s-4 r-5$ & $0 \leq r \leq s-1$ \\
$* 8 s+8$ & $12 s+9$ & $4 s+1$ & $\cdots \cdots$ \\
$10 s+r+8$ & $14 s-r+5$ & $4 s-2 r-3$ & $0 \leq r \leq 2 s-5$ \\
$16 s+9$ & $16 s+14$ & 5 & $\cdots \cdots$ \\
$16 s+6$ & $16 s+10$ & 4 & $\cdots$ \\
$16 s+8$ & $16 s+11$ & 3 & $\cdots$ \\
$14 s+7$ & $14 s+9$ & 2 & $\cdots$ \\
\hline
\end{tabular}


To complete the proof we list below the hooked $(2 n-2)$-extended $m$-nearSkolem sequence of order $n=7$ and $m=2,6$ :

$7,5,1,1,6,3,5,7,3,4,6,0,0,4$

$7,5,3,1,1,3,5,7,2,4,2,0,0,4$

The sequences presented in the following Corollaries are a result of the sequences in Theorems 13 and 14 reversed.

Corollary 1 A 3-extended $m$-near-Skolem sequence of order $n$ and defect $m$ exists if and only if one of the following is true:

1. $n \equiv 0,1(\bmod 4)$ and $m$ is odd

2. $n \equiv 2,3(\bmod 4)$ and $m$ is even

The cases $n=1$ and $(n, m)=(3,2)$ are exceptions.

Corollary $2 A(2,3)$-extended $m$-near-Skolem sequence of order $n$ and defect $m$ exists if and only if one of the following is true:

1. $n \equiv 0,1(\bmod 4)$ and $m$ is odd

2. $n \equiv 2,3(\bmod 4)$ and $m$ is even

The cases $n=1$ or 2 are exceptions.

\section{Constructions}

In this section we show how the sequences $\mathcal{O}_{n}^{m}$ and $\mathcal{T}_{n}^{m}$ can be used to construct other generalizations of Skolem sequences.

Corollary 3 When $n \equiv 0,1(\bmod 4)$, the existence of $\mathcal{O}_{n}^{m}$ and/or $\mathcal{T}_{n}^{m}$ implies the existence of $P^{2}(1, n) / m$.

Proof: A hooked 2n-extended Skolem sequence of order $n$ can be hooked together with $\mathcal{O}_{n}^{m}$ to form $P^{2}(1, n) / m$ and a $(2 n-1)$-extended Skolem sequence of order $n$ can be hooked together with $\mathcal{T}_{n}^{m}$ to form $P^{2}(1, n) / m$.

Corollary 4 For admissible $n$ and $k$, the existence of $\mathcal{O}_{n}^{m}$ implies the existence of

1. $P^{2}(1, n) / m-\{k, 4 n-2\}$

2. $P^{2}(1, n) / m-\{3,4 n+1-k\}$ 
3. $P^{2}(1, n) / m-\{2 n-3,2 n-1+k\}$

4. $P^{2}(1, n) / m-\{2 n+2-k, 2 n+4\}$

5. $P^{2}(1, n) / m-\{4 n-3\}$

6. $P^{2}(1, n) / m-\{3\}$

7. $P^{2}(1, n) / m-\{2 n-3\}$

8. $P^{2}(1, n) / m-\{2 n+3\}$

Proof: Given $\mathcal{O}_{n}^{m}$, we can construct a $k$-extended Skolem sequence of order $n$ and append this to the beginning [end], giving us (1) [(3)]. We can then reverse the resulting sequence to give us (2) [(4)]. Or we can construct a Skolem sequence of order $n$ and append it to the beginning [end] of $\mathcal{O}_{n}^{m}$ to get (5) $[(7)]$. Reversing the resulting sequence then gives us (6) [(8)].

Corollary 5 For admissible $n$ and $k$, the existence of $\mathcal{T}_{n}^{m}$ implies the existence of

1. $P^{2}(1, n) / m-\{k, 4 n-1,4 n\}$

2. $P^{2}(1, n) / m-\{2,3,4 n+2-k\}$

3. $P^{2}(1, n) / m-\{2 n-2,2 n-1,2 n+k\}$

4. $P^{2}(1, n) / m-\{2 n+2-k, 2 n+3,2 n+4\}$

5. $P^{2}(1, n) / m-\{4 n-2,4 n-1\}$

6. $P^{2}(1, n) / m-\{2,3\}$

7. $P^{2}(1, n) / m-\{2 n-2,2 n-1\}$

8. $P^{2}(1, n) / m-\{2 n+2,2 n+3\}$

Proof: Given $\mathcal{T}_{n}^{m}$, we can construct a $k$-extended Skolem sequence of order $n$ and append this to the beginning [end], giving us (1) [(3)]. We can then reverse the resulting sequence to give us (2) [(4)]. Or we can construct a Skolem sequence of order $n$ and append it to the beginning [end] of $\mathcal{T}_{n}^{m}$ to get (5) $[(7)]$. Reversing the resulting sequence then gives us (6) [(8)]. 


\section{Conclusions}

In this paper we showed that the necessary conditions for the existence of $(2 n-3)$-extended $m$-near-Skolem sequences and hooked $(2 n-2)$-extended Skolem sequences are also sufficient. We then used these sequences in the construction of other generalized Skolem sequences.

Skolem sequences and their generalizations are extremely useful in constructing various combinatorial designs and thus any and all existence results for other generalizations are welcome. Two welcome results would be on the general case for $k$-extended $m$-near-Skolem sequences and $(p, q)$-extended $m$ near-Skolem sequences (an $m$-near Skolem sequence with two hooks). Another interesting generalization would be $\left(m_{1}, m_{2}\right)$-near Skolem sequences, where there are two defects, $m_{1}, m_{2}$, as opposed to one. We conjecture that the necessary conditions for the existence of these three sequences are also sufficient. We also note that we have tested these sequences up to large values of $n$ and the conjectures hold. Although these conjectures are not in the literature (to the best of our knowledge), some have been mentioned in several conferences.

Conjecture $1 A k$-extended $m$-near-Skolem sequence of order $n$ exists if and only if one of the following is true:

1. $n \equiv 0,1(\bmod 4) m$ and $k$ are of the same parity

2. $n \equiv 2,3(\bmod 4)$ and $m$ and $k$ are of opposite parity

Conjecture $2 A(p, q)$-extended $m$-near-Skolem sequence of order $n$ exists if and only if one of the following is true:

1. $n \equiv 0,1(\bmod 4), m$ even, and $p$ and $q$ are of the same parity

2. $n \equiv 0,1(\bmod 4), m$ odd, and $p$ and $q$ are of opposite parity.

3. $n \equiv 2,3(\bmod 4), m$ odd, and $p$ and $q$ are of the same parity

4. $n \equiv 2,3(\bmod 4), m$ even, and $p$ and $q$ are of opposite parity.

Conjecture $3 A\left(m_{1}, m_{2}\right)$-near-Skolem sequence of order $n$ exists if and only if one of the following is true:

1. $n \equiv 0,1(\bmod 4)$ and $m_{1}$ and $m_{2}$ are of the same parity

2. $n \equiv 2,3(\bmod 4)$ and $m_{1}$ and $m_{2}$ are of opposite parity.

The construction of these sequences seem difficult at this point and require other methods than those presented in the paper. 


\section{References}

[1] J. Abrham and A. Kotzig, Skolem sequences and additive permutations, Discrete Math. 37 (1981) 143 - 146.

[2] C.A. Baker, Extended Skolem Sequences, J. Combin. Designs 3 (1995), $363-379$.

[3] C.A. Baker, N. Shalaby, A. Sharary, and R.J. Nowakowski, M-fold and extended m-fold Skolem sequences, Utilitas Mathematica 45 (1994), pp. $153-167$.

[4] E. Billington, Cyclic balanced ternary designs with block size three and index two, Ars. Combin. (1987) 215 - 232.

[5] C. Dudley Langford, Problem, Math. Gaz., 42 (1958), 228.

[6] V. Linek and Z. Jiang, Extended Langford Sequences with Small Defects, Journal of Combinatorial Theory, Series A 84, 38 - 54 (1998)

[7] V. Linek and Z. Jiang, Hooked k-extended Skolem sequences, Discrete Math. 196 (1999) $229-238$.

[8] V. Linek and N. Shalaby, The existence of $(p, q)$-extended Rosa sequences, Preprint, 35 pages.

[9] E. Morgan, Balanced ternary designs with block size three, Combinatorial Math. VII, Lecture Notes in Math. 829, Springer-Verlag, Berlin, New York, 1980, $186-198$.

[10] R.S. Nickerson, Problem E1845, Amer. Math. Monthly 73 (1966), 81; Solution, 74 (1974), $591-592$.

[11] G. Nordh, Perfect Skolem Sets, Preprint, 2005.

[12] E.S. O'Keefe, Verification of a conjecture of Th. Skolem, Math. Scand. 9 (1961) $80-82$.

[13] C.J. Priday, On Langford's problem, I, Math. Gaz., 433 (1959), 250 - 253.

[14] A. Rosa, Poznamka o cyklikch Steinerovych systemoch trojic (a note on cyclic Steiner triple systems(Slovak)), Mat. Fyz. Casopis 16 (1966) $285-$ 290.

[15] N. Shalaby, The existence of near-Rosa and hooked near-Rosa sequences, Discrete Math. 261 (2003) 435 - 450. 
[16] N. Shalaby, The existence of near-Skolem and hooked near-Skolem sequences, Discrete Math. 135 (1994) 303 - 319.

[17] N. Shalaby, Skolem Sequences, in: C. Colbourn, J. Dinitz (Eds.), The CRC Handbook of Combinatorial Designs, CRC Press, Boca Raton, 1996 pp. $457-461$

[18] N. Shalaby and M.A. Al-Gwaiz, Generalized, Hooked, Extended, and Near-Skolem Sequences, JCMCC 26 (1998), pp. 113 - 128.

[19] N. Shalaby and T. Stuckless, The Existence of Looped Langford Sequences, Crux Mathematicorum, 26 (2000), No.2, 86 - 92.

[20] J.E. Simpson, Langford Sequences: Perfect and Hooked, Discrete Math., 44 (1983), $97-104$.

[21] Th. Skolem, On certain distributions of integers in pairs with given differences, Math. Scand. 5 (1957) 57 - 58.

[22] R.G. Stanton and I.P. Goulden, Graph factorization, general triple systems and cyclic triple systems, Aequationes Mat. 22 (1981), $1-28$.

\section{Received: October 4, 2005}

